\title{
A BRIEF DERIVATION OF THE HEISENBERG COMMUTATION RELATIONS
}

\author{
REESE T. PROSSER
}

\begin{abstract}
The commutation relations for the canonical coordinates of a mechanical system are here derived from a suitably defined form of translational invariance.
\end{abstract}

Let us assume that we are dealing with a physical system which can be adequately described in terms of a finite set of canonical coordinates, $\left(x_{1}, \cdots, x_{n}\right)$, whose expected values in every admissible state of the system are given by a positive linear functional on the moment algebra $A\left(x_{1}, \cdots, x_{n}\right)$ generated by the $x_{i}$ over the complex field $C[1]$. We may take for $A$ the algebra $T$ of all noncommutative polynomials with complex coefficients in the $x_{i}$ modulo the ideal $J$ of relations on which all expectations vanish.

Let us introduce, for each real $a \in C$, the translation (or virtual displacement) $\tau_{j}(a)$ of the $j$ th coordinate by the formula

$$
\begin{aligned}
\tau_{j}(a) x_{i} & =x_{j}+a & & \text { if } i=j, \\
& =x_{i} & & \text { otherwise. }
\end{aligned}
$$

Each such translation assigns to every polynomial $p$ in the $x_{i}$ a new polynomial $\tau_{j}(a) p$ obtained by replacing $x_{j}$ by $x_{j}+a$ throughout.

Let us now assume that the ideal $J$ defining our moment algebra $A$ satisfies two natural requirements:

(a) if $p \equiv 0 \bmod J$, then $\tau_{j}(a) p \equiv 0 \bmod J$, for all $j$ and all $a$. This says that $J$ is stable under translations, and hence that $A$ admits these translations as automorphisms.

(b) If $\tau_{j}(a) p \equiv p \bmod J$ for all $a$, then $p \equiv q \bmod J$, where $q$ is independent of $x_{j}$.

This says that any polynomial invariant, $\bmod J$, under $\tau_{j}(a)$ for all $a$ can be expressed, $\bmod J$, as a polynomial in the $x_{i}$ for $i \neq j$.

Both of these requirements appear to be essential to any variational formulation of the laws of motion of the system.

Let us call any moment algebra whose defining ideal of relations satisfies these two requirements acceptable. It is easy to verify that the moment algebras associated with the systems of both classical and

Received by the editors May 23, 1969 and, in revised form, March 10, 1970.

AMS 1969 subject classifications. Primary 8122.

Key words and phrases. Quantum mechanics, canonical coordinates, commutation relations. 
quantum mechanics are acceptable, and so is any tensor product of the two. We assert here that these are the only possibilities:

THEOREM. Every acceptable moment algebra is either a classical moment algebra, a quantum moment algebra, or a tensor product of the two.

Proof. Consider the commutator $\left[x_{i}, x_{k}\right]=x_{i} x_{k}-x_{k} x_{i}$. This polynomial is evidently invariant under $\tau_{j}(a)$ for all $j$ and all $a$. If $A$ is acceptable, then $\left[x_{i}, x_{k}\right]$ is independent, $\bmod J$, of $x_{j}$ for $a l l j$, and hence must be a constant, $\bmod J$. If we put $\left[x_{i}, x_{k}\right] \equiv b_{i k}$, then the $b_{i k}$ form a skew-symmetric matrix of complex numbers. It follows by standard methods that we can choose linear combinations $\left(y_{1}, \cdots\right.$, $y_{n}$ ) of the $x_{i}$ such that, for some $m \leqq n / 2$,

$$
\left[y_{i}, y_{k}\right] \equiv\left\{\begin{aligned}
1 & \text { if } 1 \leqq i=k-m \leqq m \\
-1 & \text { if } m+1 \leqq i=k+m \leqq 2 m \\
0 & \text { otherwise }
\end{aligned}\right\} \quad \bmod J
$$

In particular, if $m=0$, we have that the $y_{i}$ all commute, while if $m=n / 2$, we have that the $y_{i}$ satisfy the Heisenberg commutation relations.

Let $K$ denote the ideal generated in $T$ by these commutation relations, and note that $K \subset J$. It remains to show that $K=J$. In any case it follows from the commutation relations that the $x_{i}$, together with 1 , form the basis of a nilpotent Lie algebra in $A$, and that $A$ can be realized as a homomorphic image of the associated universal enveloping algebra $U=T \bmod K$. Now we know from the BirkhoffWitt theorem that every polynomial has a unique expression, $\bmod K$, as a linear combination of monomials of the form $x_{1}^{d_{1}} \cdots x_{n}^{d_{n}}$. If such a polynomial $p$ lies in $J$, then so do all its translates; moreover, if $p$ is of degree $d_{j}$ in $x_{j}$ then evidently $\tau_{j}(a) p-p$ is of degree $d_{j}-1$ in $x_{j}$ for all $a \neq 0$. From these observations we infer that if $J \neq K$, then $J$ contains the constant polynomials, an unacceptable alternative.

\section{REFERENCES}

1. R. T. Prosser, A new formulation of particle mechanics, Mem. Amer. Math. Soc. No. 61 (1966). MR 32 \#8545.

Dartmouth College, Hanover, New Hampshire 03755 